\title{
Article
}

\section{Whole-body physiology-based pharmacokinetics of caspofungin for general patients, intensive care unit patients and hepatic insufficiency patients}

\author{
Qian-ting YANG ${ }^{1}$, Ya-jing ZHAI ${ }^{1}$, Lu CHEN ${ }^{1}$, Tao ZHANG ${ }^{1}$, Yan YAN ${ }^{1}$, Ti MENG ${ }^{1}$, Lei-chao LIU ${ }^{1}$, Li-mei CHEN ${ }^{2}$, Xue WANG ${ }^{3}$, Ya- \\ lin DONG ${ }^{1, *}$ \\ ${ }^{1}$ Department of Pharmacy, The First Affiliated Hospital of Xi'an Jiaotong University, Xi'an 710061, China; ${ }^{2}$ Department of Hematology, \\ The First Affiliated Hospital of Xi'an Jiaotong University, Xi'an 710061, China; ${ }^{3}$ Central Intensive Care Unit, The First Affiliated Hospital \\ of Xi'an Jiaotong University, Xi'an 710061, China
}

\begin{abstract}
Caspofungin is an echinocandin antifungal agent licensed as a first-line therapy for invasive candidiasis in patients with moderate to severe illness or recent exposure to azoles. In this study we developed a whole-body physiology-based pharmacokinetics (WB-PBPK) model to predict the pharmacokinetics (PK) of caspofungin, and combined with Monte Carlo simulation (MCS) to optimize clinical dosage regimens of caspofungin in different kinds of patients. A WB-PBPK model of caspofungin was built and validated with raw data from 4 previous trials of general patients, intensive care unit (ICU) patients with Child-Pugh B, ICU patients on continuous renal replacement therapy, mild and moderate hepatic insuffciency $(\mathrm{HI})$ patients. MCS was used to optimize clinical dosage regimens of caspofungin in these patients. A cumulative fraction of response (CFR) value of $\geq 90 \%$ was considered to be the minimum for achieving optimal empirical therapy. The simulated results of the WB-PBPK model were in good agreement with observed values of all trials. For general and ICU patients with caspofungin 70/50 mg, AUC and Cmax were decreased with the increase of body weight (BW) and showed great variation. MCS showed all general patients achieved CFR $\geq 90 \%$ regardless of BW. But not all ICU patients with higher BW ( $\geq 70 \mathrm{~kg}$ ) could achieve CFR $\geq 90 \%$. Compared with standard dosage regimens in general patients, caspofungin $70 / 35 \mathrm{mg}$ in ICU patients with Child-Pugh B achieved significantly decreased AUC and $C_{\max }$, but obtained similar AUC and $C_{\text {max }}$ in moderate $H I$ patients with Child-Pugh B. The WB-PBPK model of caspofungin is able to predict PK of all populations correctly. The combined WB-PBPK model with MCS can successfully optimize clinical dosage regimens of caspofungin in all patient populations.
\end{abstract}

Keywords: caspofungin; antifungal agent; whole-body physiology-based pharmacokinetics model; intensive care unit; hepatic insuffciency; cumulative fraction of response

Acta Pharmacologica Sinica (2018) 39: 1533-1543; doi: 10.1038/aps.2017.176; published online 31 May 2018

\section{Introduction}

Caspofungin is an echinocandin antifungal agent licensed as a first-line therapy for invasive candidiasis in patients with moderate to severe illness or recent exposure to azoles ${ }^{[1]}$. The recommended dosage regimen of caspofungin is a loading dose of $70 \mathrm{mg}$ followed by $50 \mathrm{mg}$ daily (70/50 mg), administered intravenously over $1 \mathrm{~h}$. Caspofungin is highly protein bound $(96 \%)$ and metabolizes slowly in the liver ${ }^{[2-4]}$. Its liver uptake is a biphasic process and its binding to the surface of hepatocytes is fast and reversible. Studies demonstrated

\footnotetext{
* To whom correspondence should be addressed.

E-mail dongyalin@mail.xjtu.edu.cn

Received 2017-07-30 Accepted 2017-11-26
}

that the uptake of caspofungin by liver is related to the active organic anion transporting polypeptide 1B1 (OATP1B1) ${ }^{[5]}$. The plasma clearance (CL) of caspofungin is 10 to $12 \mathrm{~mL} / \mathrm{min}^{[2] . ~ I t ~}$ is eliminated mainly by hepatic, for only one to two percent of the antifungal agent is through renal clearance ${ }^{[6]}$. The elimination of caspofungin from plasma is slow and the half-life of caspofungin is 9-11 $\mathrm{h}^{[2]}$.

Clinically, pharmacokinetics (PK) parameters in intensive care unit (ICU) patients are often different from those in healthy subjects ${ }^{[7]}$. Factors associated with alterations in PK include changes in organ function, use of extracorporeal clearance techniques and drug interactions ${ }^{[8,9]}$. It was also reported that caspofungin plasma concentrations were influenced by hypoalbuminaemia and hepatic impairment $(\mathrm{HI})^{[7]}$. One study demonstrated that caspofungin trough plasma concentrations 
$\left(C_{\min }\right)$ ranges were relatively wide in surgical ICU patients and influenced by protein binding ${ }^{[7]}$. Another study showed that caspofungin area under the curve (AUC) was increased by $55 \%$ and $76 \%$ in patients with mild and moderate $\mathrm{HI}$, respectively ${ }^{[4,10]}$. But at present, data are very limited and absent on these special patients, an increase in knowledge on these patients should be considered. Clinically, patients weighing $>80 \mathrm{~kg}$ are advised to receive a dosage regimen of $70 / 70$ $\mathrm{mg}^{[11]}$. But studies of general ICU population and hematopoietic stem cell transplantation patients found that body weight (BW) (range 50-99 kg) had no effect on caspofungin $\mathrm{PK}^{[9,12]}$. Whether age and race could influence the PK of caspofungin is not clear. On the other hand, one study demonstrated that the vast majority of fungal infections are caused by Candida $\mathrm{spp}^{[9]}$. In the critically ill, the infections of invasive Candida spp. are associated with high crude and attributable mortalities as high as $60 \%$ and $40 \%$, respectively ${ }^{[13]}$. One multicentre observational study in Spain had carried out that mortality was associated with age, Candida spp., different from C. parapsilosis and inadequate treatment ${ }^{[14]}$. All of these ask for a model which could predict the PK of caspofungin in different populations (general patients, ICU patients and HI patients) with different physical conditions, BW, age, race, Candida spp. And based on the PK parameters, a Monte Carlo simulation (MCS) could be used to optimize the clinical dosage regimens of caspofungin in different patient populations.

Whole-body physiology-based PK (WB-PBPK) modellings consider the physical and chemical characteristics of a drug and it also give thorough consideration of physiological processes of drug absorption, distribution, metabolism and elimination accurately ${ }^{[15]}$. Based on a series of parameters, such as physiological and physicochemical properties, CL, distribution into tissues and metabolism or active transport, WBPBPK could predicts PK of a drug ${ }^{[15]}$. Then based on a series of equations, we can develop a WB-PBPK model which could be applied to forecast disease dependencies ${ }^{[7,9]}, \mathrm{BW}$, age, and race can also be used to investigate the variability expected in different patient populations ${ }^{[6,12]}$.

The aim of the present study was to build and verify a WBPBPK model which could predict the PK of caspofungin in general patients, ICU patients with Child-Pugh B, ICU patients on continuous renal replacement therapy (CRRT) and HI patients, then to evaluate the impact on the PK of caspofungin between different individuals through stochastic simulations of different body weight, age and race using the validated WBPBPK model. Finally, combined with MCS technique to help in the selection of a safe and effective administration scheme after simulation of the potential exposure and disposition in different patient populations.

\section{Materials and methods PK data}

Clinical PK data for intravenous administration of caspofungin in healthy adults and all patients were obtained from the literature $^{[4,9,10,16,17]}$. Raw data of 20 healthy subjects, receiving single intravenous doses of $20 \mathrm{mg}$, $40 \mathrm{mg}$, $70 \mathrm{mg}$, $100 \mathrm{mg}$, respectively, were used for model development. The mean age was 29 years (range, 21-39 years) and the mean weight was $75 \mathrm{~kg}$ (range, $60-88 \mathrm{~kg}$ ). The observed mean concentrations of caspofungin over time were from the literature ${ }^{[4]}$. Mean concentrations of caspofungin over time from a doubleblind, placebo-controlled, serial-panel study of 24 healthy male subjects to investigate the pharmacokinetics of caspofungin following daily doses of 15,35 , or $70 \mathrm{mg}$ were used to validate the model ${ }^{[4]}$. Another trial of caspofungin in healthy subjects studied the standard dosage regimen of $70 / 50 \mathrm{mg}^{[10]}$. Plasma drug profiles defined by concentrations at $0 \mathrm{~h}$ (predose) and at $0.5,0.75,1,1.25,1.5,2,4,8,12$, and 24 h postdose were obtained on days 1 and 14. Intervening trough (24-h postdose) concentrations $\left(C_{\text {min }}\right)$ were obtained prior to dosing on $\mathrm{d} 3,4,7$, and $10^{[4]}$. The relevant data are shown in Table 1.

To develop and qualify the WB-PBPK model in general patients ${ }^{[16]}$, ICU patients with Child-Pugh $\mathrm{B}^{[9]}$ and ICU patients on CRRT ${ }^{[17]}$, HI patients ${ }^{[10]}$, raw data from 4 clinical trials were used. The study description, population included, treatment schedule, doses and sampling scheme are shown in Table 1. The study of general patients was a formal phase II dose escalation trial in patients with invasive aspergillosis. Caspofungin was administered once daily as an iv infusion over $120 \mathrm{~min}$ at $70 \mathrm{mg}$ (9 patients), $100 \mathrm{mg}$ (8 patients), $150 \mathrm{mg}$ (9 patients) and $200 \mathrm{mg}$ (20 patients). Pharmacokinetic sampling was performed serially on $\mathrm{d} 1(2,3,5-7$ and $24 \mathrm{~h}$ after the infusion started) and peak (at the end of infusion) and trough levels (immediately before the next infusion started) were collected on $\mathrm{d} 4,7,14$ and 28 . All observed data from pharmacokinetic sampling were pooled as mean \pm standard deviation ${ }^{[16]}$. Depending on the WB-PBPK model, we studied the recommended dosage regimens $(70 / 50 \mathrm{mg}$ in ICU patients, mild $\mathrm{HI}$; $70 / 35 \mathrm{mg}$ in moderate HI patients) of caspofungin ${ }^{[9,10,17]}$. To evaluate the model, we collected 508 trough concentrations from 161 patients (39 ICU patients with Child-Pugh B, 16 ICU patients on CRRT, 47 moderate $\mathrm{HI}$ patients and 68 mild HI patients) of the First Affiliated Hospital of Xi'an Jiaotong University. The pharmacokinetic sampling were pooled as mean \pm standard deviation in ICU patients and HI patients. The study was approved by the Ethics Committee of the First Affiliated Hospital of $\mathrm{Xi}^{\prime}$ an Jiaotong University. All subjects signed the informed consent before any screening item being performed.

\section{Model development}

The WB-PBPK model of caspofungin, which was built by GastroPlus version 9.0 (Simulations Plus, USA), was used for all of the simulations in healthy subjects. The WB-PBPK (disposition) model was composed of a number of tissue compartments which were linked together by venous and arterial blood circulation. These compartments include the heart, lung, brain, adipose, muscle, skin, liver, kidney, and so on. The drug distribution into different compartments was driven by perfusion-limited kinetics for all of the tissues were considered to be well-stirred compartments. Each compartment was defined by an associated tissue blood flow rate, volume and a tissue to-plasma partition coefficient. We used the built-in 


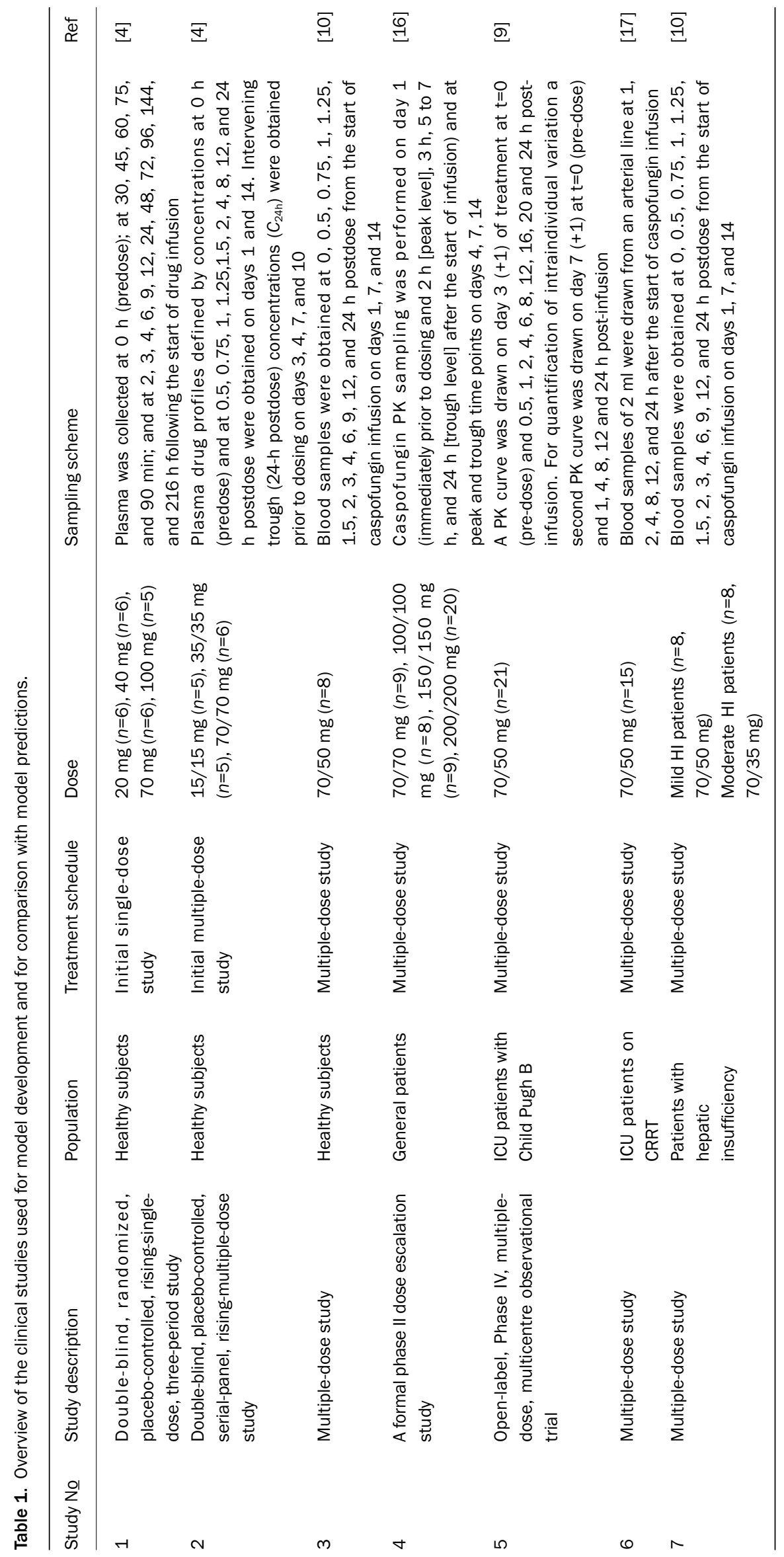


mass balance differential equations to develop the model ${ }^{[18]}$.

Vital physicochemical parameters and the key vitro data for caspofungin are depicted in Table 2. The $\mathrm{p} K_{\mathrm{a}}, \mathrm{Rbp}$, solubility$\mathrm{pH}$, effective permeability and particle size were obtained using the ADMET Predictor ${ }^{\mathrm{TM}}$ module of GastroPlus. Human $\log P$, fup (plasma unbound drug) was obtained from the related literature ${ }^{[19,20]}$. The tissue-to-plasma partition coefficients of caspofungin were calculated using established tissuecomposition based models. Total plasma CL of caspofungin was calculated by the PKplus model of the GastroPlus version 9.0 (Table 3). The results of non-compartment model will be used to develop the WB-PBPK model. The main elimination route of caspofungin is hepatic (98\%) and only two percent of caspofungin is through renal clearance. So the CL data of liver and renal used in the model will based on this. The simulation time was $144 \mathrm{~h}$ and the volume of dosage administration was $250 \mathrm{~mL}$.

\section{PK simulations}

Based on the raw data from 4 clinical trials, we developed and qualified the WB-PBPK model in general patients, ICU patients with Child-Pugh B and ICU patients on CRRT and HI patients. The study description, population included, treatment schedule, doses and sampling scheme were recorded and used in the development and verification of the WB-PBPK models. Total plasma CL from different populations were calculated by the PKplus model of the GastroPlus version 9.0 (Table 3). The results of non-compartment model were used to develop the WB-PBPK model. The simulation time were 14 $\mathrm{d}$ and the volume of dosage administration were $250 \mathrm{~mL}$.

\section{Model evaluation}

To evaluate the WB-PBPK models, a visual comparison between the predicted data of GastroPlus version 9.0 and the observed data (literature resources and our research data) of the different trials from different populations was carried out. Goodness of fit (GOF) plots were performed to analyze the accuracy between simulated and observed data. The prediction error (PE) was estimated according to Eq. $1^{[19]}$.
$\mathrm{PE}=($ simulated-observed $) /$ observed $\times 100$

The fold-error, which represents the difference between observed and predicted in vivo values, was used to assess the accuracy of the predicted PK parameters, and if the fold-error was less than two, the prediction was considered successful ${ }^{[21,22]}$.

Fold-error $=$ observed $/$ predicted (observed value $>$ predicted value)

(Eq.2)

Fold-error=predicted $/$ observed (observed value $<$ predicted value)

(Eq.3)

\section{Model application}

\section{Virtual trial simulations}

For general and ICU patients, we simulated the recommended dosage regimens $(70 / 50 \mathrm{mg})$ of caspofungin. On the other hand, other two multiple dosage regimens $(70 / 35 \mathrm{mg}, 70 / 70$ $\mathrm{mg}, 100 / 100 \mathrm{mg}$ ) of caspofungin were simulated to investigate the effects of different dosage regimens of caspofungin in these patients.

For HI patients, except the recommended dosage regimens (70/35 mg for moderate HI patients, 70/50 $\mathrm{mg}$ for mild HI patients), dose reduction of 50/25 $\mathrm{mg}$ and dose escalating of $70 / 70 \mathrm{mg}, 100 / 100 \mathrm{mg}$ were simulated using the WB-PBPK model.

PK variability of varying body size of the recommended dosage regimens of caspofungin in general patients, ICU and $\mathrm{HI}$ patients were assessed by creating virtual populations weighing $40-100 \mathrm{~kg}$. Virtual ages of 18-95 years were simulated in these patients, too. What's more, different race groups, representatives of Asian and Westerner were simulated. Based on the WB-PBPK model, we could obtain the PK parameters including peak plasma concentrations $\left(C_{\max }\right), C_{\min }$ AUC and CL of these patients under different weight conditions.

\section{Dosage optimization}

To describe caspofungin pharmacokinetic/pharmacodynamics (PK/PD) adequacy for the treatment of invasive Candida spp. and determine whether the different dosage regimens

Table 2. Physicochemical and in vitro data for caspofungin used in the simulations.

\begin{tabular}{|c|c|c|}
\hline Parameter & Value & Source \\
\hline Molecular weight & 1093.3 & Estimated by ADMET Predictor ${ }^{\mathrm{TM}}$ \\
\hline $\log P$ & 0 & {$[25]$} \\
\hline Solubility $(\mathrm{mg} / \mathrm{mL})$ & 18.58 & Estimated by ADMET Predictor ${ }^{\mathrm{TM}}$ \\
\hline $\mathrm{pH}$ for solubility & 10.25 & Estimated by ADMET Predictor ${ }^{\mathrm{TM}}$ \\
\hline Effective permeability $\left(P_{\text {eff }}, \mathrm{cm} / \mathrm{s}\right)$ & 171 & Estimated by ADMET Predictor ${ }^{\mathrm{TM}}$ \\
\hline Diff. Coeff $\left(\mathrm{cm}^{2} / \mathrm{s}\right)$ & $3.4 \times 10^{4}$ & Estimated by ADMET Predictor ${ }^{\mathrm{TM}}$ \\
\hline Drug particle density $(\mathrm{g} / \mathrm{mL})$ & 1.2 & Estimated by ADMET Predictor ${ }^{\mathrm{TM}}$ \\
\hline Fup (Plasma unbound drug, \%) & 3 & {$[26]$} \\
\hline Rbp (Blood/Plasma concentration ratio) & 0.55 & Estimated by ADMET Predictor ${ }^{\mathrm{TM}}$ \\
\hline
\end{tabular}




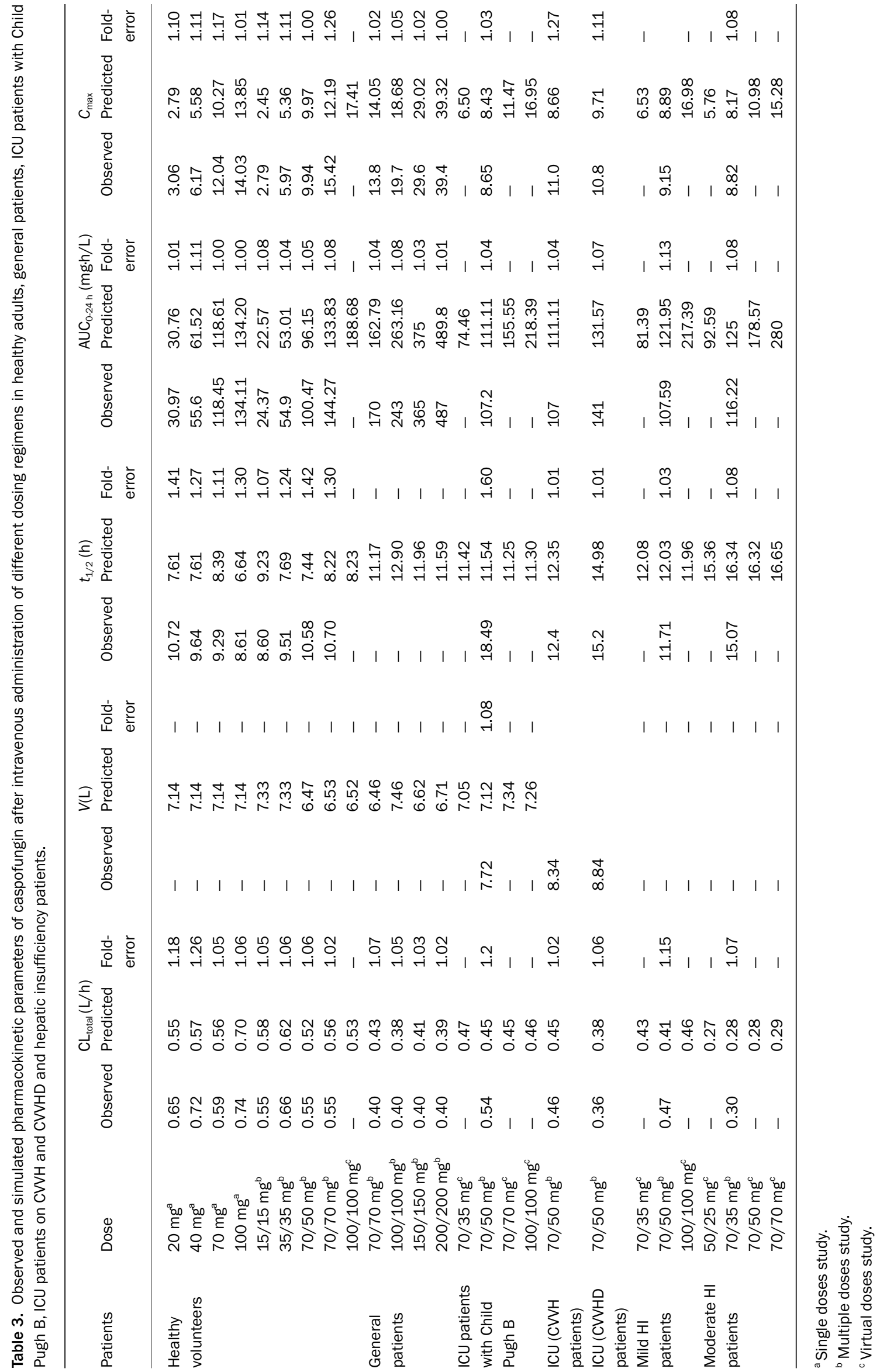


of caspofungin could achieve PK/PD targets in the general patient populations, ICU and HI patients. A MCS method was performed using the simulated data of WB-PBPK model. Through virtual simulation of Gastroplus, we obtained the AUC of different dosage regimens of these special patients. The MCS methods, the pharmacodynamic targets for Candida species $\left(\mathrm{AAUC}_{0-24} / \mathrm{MIC}\right.$ ratio for $C$. albicans, C. glabrata and $C$. parapsilosis) and the minimum inhibitory concentration distributions for Candida species have been described previously ${ }^{[23]}$. The optimal dosage regimens were evaluated to compare the simulated probability of target attainment (PTA) and cumulative fraction of response (CFR) in these subjects. A CFR value of $\geq 90 \%$ was considered to be an appropriate empirical dosage regimen.

\section{Results}

\section{Caspofungin PK}

The WB-PBPK model for caspofungin was established according to the scheme of relevant tissue compartments of the disposition model. Simulations were conducted based on the physicochemical and in vitro data of caspofungin (Table 2). Hepatic and renal caspofungin CL (Table 3) were calculated by the PKplus model, as described in the method section.

The observed (literate resources data) and mean simulated plasma concentration-time profiles of caspofungin in healthy subjects are vividly presented in Figure 1 . The accuracy of the predictions are summarized in Table 3. The predicted PK parameters were nearly consistent $(<2$-fold error) with the

A

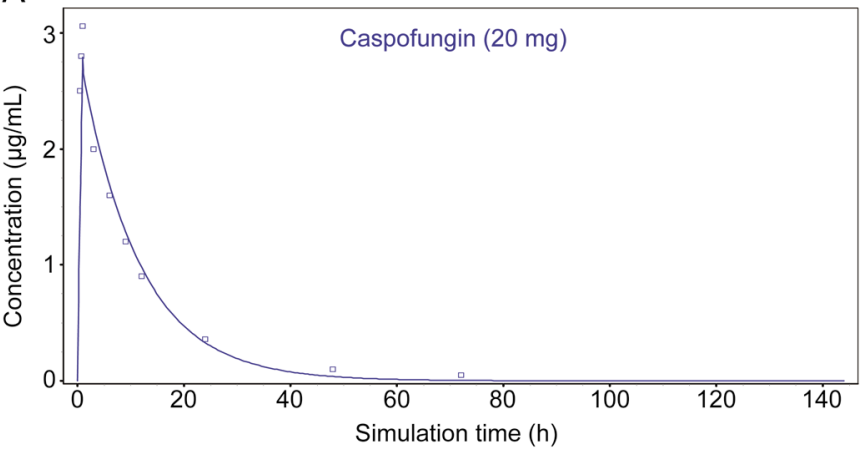

B

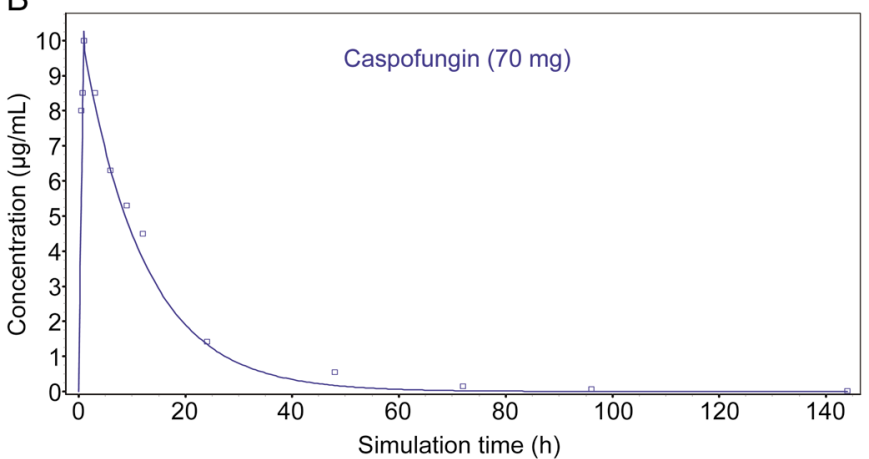

observed values (literate resources data). Accurately, using the in silico data, in vitro data, WB-PBPK model could simulate the plasma concentration-time profile following intravenous administration and predicted CL as inputs to the model, as shown in Figure 1 and Table 3.

\section{Data verification}

The established WB-PBPK model of caspofungin could predict the observed concentration-time profile (literate resources data) for all dose groups of different populations accurately (Figures 1-3, Supplementary Figure 1). For all doses levels, the simulated peak concentrations seem to be underrated by $20 \%$ but they were within the error range. As shown in Supplementary Figure 1, the predicted concentration-timecurve was in accordance with the observed mean values (literate resources data) of healthy subjects with the multiple doses administration of $15 / 15 \mathrm{mg}, 35 / 35 \mathrm{mg}, 70 / 50 \mathrm{mg}$ and $70 / 70 \mathrm{mg}$, which is the same to general patients with the dosage regimens of $70 / 70 \mathrm{mg}, 100 / 100 \mathrm{mg}, 150 / 150 \mathrm{mg}$ and 200/200 mg (Figure 2). According to the GOF plot, we could see a good accuracy between predicted and observed data (literate resources data, Supplementary Figure 2A-B). Only the simulated peak concentrations were undervalued in Gastroplus. For ICU patients with Child-Pugh B, ICU patients on CRRT and HI patients, when using the WB-PBPK distribution model to simulate plasma concentrations, a reasonable match to the measured concentration range was observed (Figure 3). The GOF plot showed a good accuracy between predicted

B

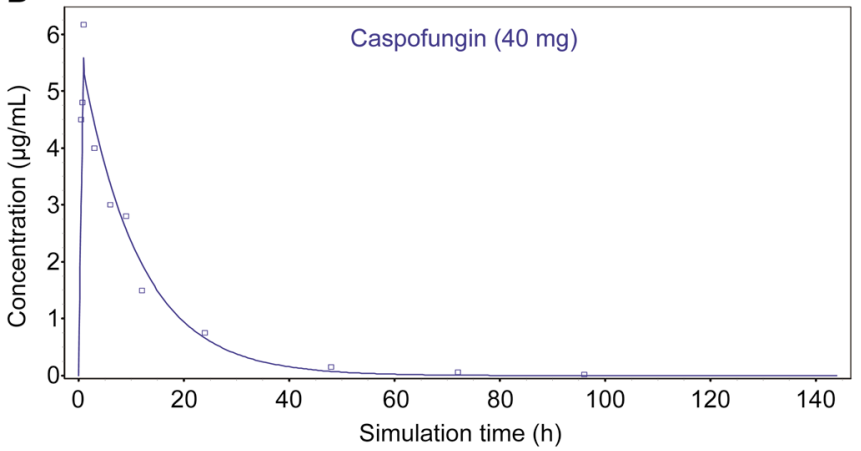

D

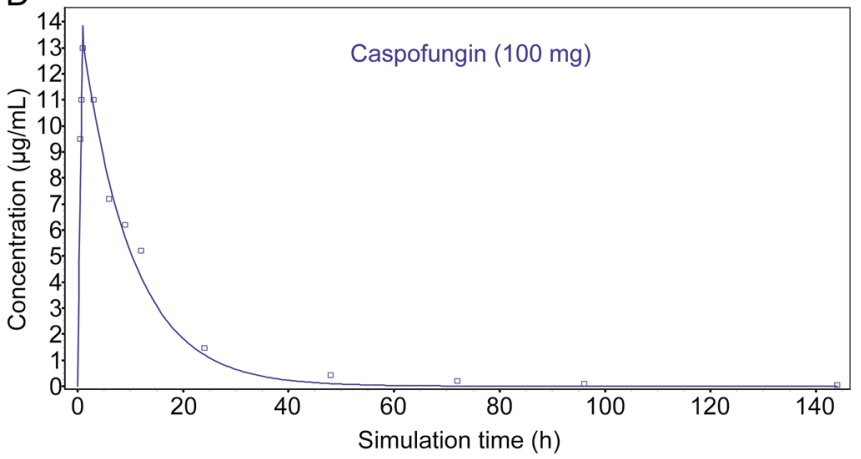

Figure 1. Simulated (line) and observed ${ }^{[4]}$ (square) plasma concentration-time profiles of caspofungin after $20 \mathrm{mg}(\mathrm{A}), 40 \mathrm{mg}(\mathrm{B}), 70 \mathrm{mg}(\mathrm{C})$ and $100 \mathrm{mg}$ (D) single dose intravenous administration in healthy subjects. 

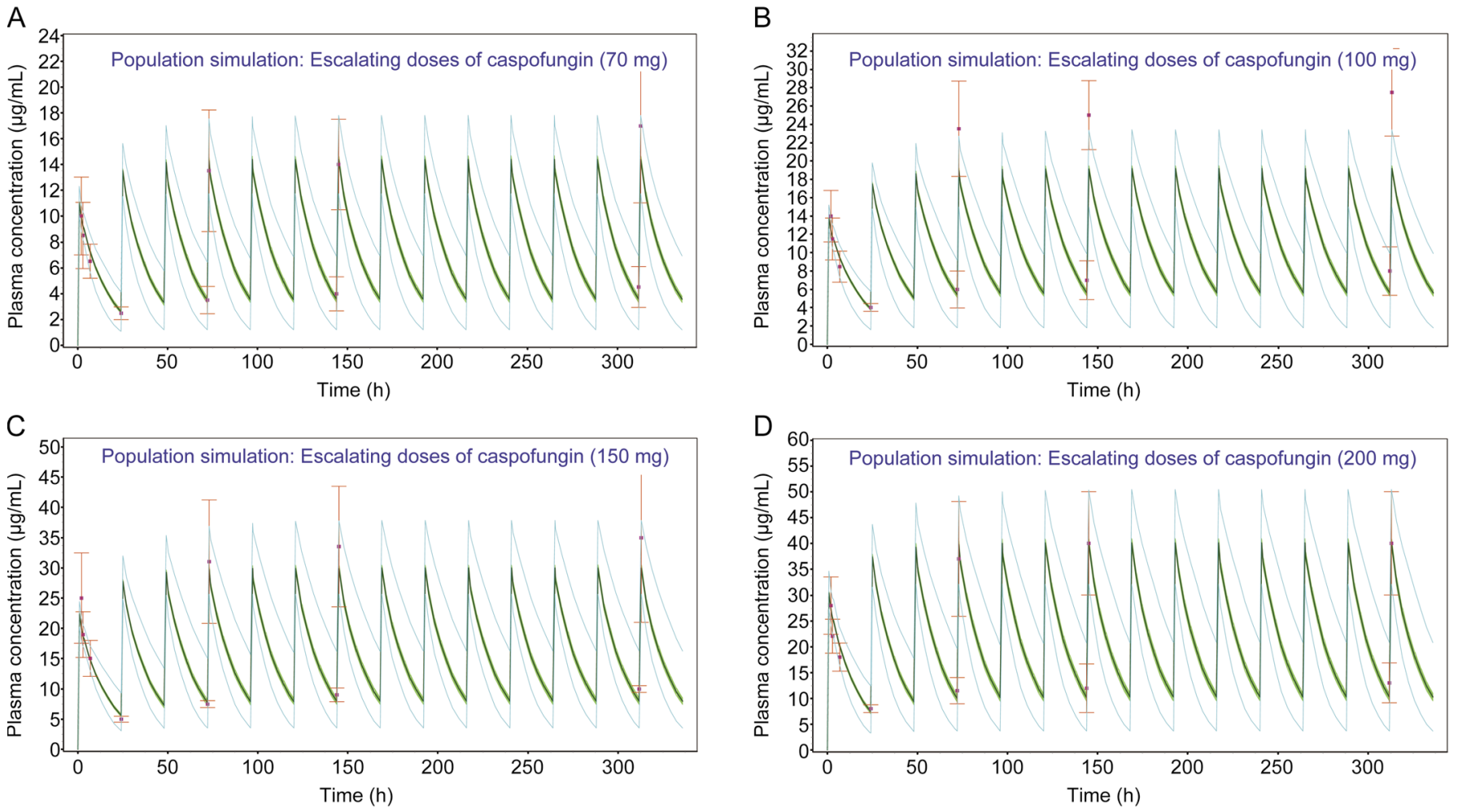

Figure 2. Simulated and mean observed (symbol $\pm S D)^{[16]}$ plasma concentration-time profiles of caspofungin after 70/70 mg (A), 100/100 mg (B), $150 / 150 \mathrm{mg}(C)$ and $200 / 200 \mathrm{mg}(\mathrm{D})^{[16]}$ intravenous multiple doses in general patients. A solid thick line adjacent to the middle of the concentrationtime profile represents the mean of the predictive values. Solid squares represent the observed clinical concentration-time data ${ }^{[16]}$. The shaded area represents the $90 \%$ confidence interval for the simulated data, and the thin lines on either hand represent individual simulated results of the $100 \%$ range of simulated individual data.

and observed data (literate resources data, Supplementary Figure 2C-D). The simulated peak levels seemed to be underestimated by $10 \%$, which were within the error range. The simulated trough levels seemed to be underestimated by $10 \%$ in ICU patients with Child Pugh B and 5\% in ICU patients on CRRT and mild HI patients compared with the observed data (literate resources and our research data), for moderate HI patients, it showed a sufficient accuracy between predicted and observed data. The CL, $V_{\mathrm{ss}}, t_{1 / 2}$, AUC, $C_{\max }$ of different patients with different dosage regimens are shown in Table 3. The fold-error of all dosage regimens were less than 2 .

\section{Virtual trial simulations}

PK parameters of general patients and ICU patients are shown in Table 3. Particularly, the PK parameters of ICU patients were recorded. At steady state, the AUC of $70 / 35 \mathrm{mg}$, 70/70 $\mathrm{mg}, 100 / 100 \mathrm{mg}$ of caspofungin in ICU patients were 74.5 $\mathrm{mg} \mathrm{h} / \mathrm{L}, 155.5 \mathrm{mg} h / \mathrm{L}, 218.4 \mathrm{mg} \mathrm{h} / \mathrm{L}$, respectively. The AUC of moderate $\mathrm{HI}$ patients were $92.6 \mathrm{mg} \cdot \mathrm{h} / \mathrm{L}, 178.6 \mathrm{mg} \cdot \mathrm{h} / \mathrm{L}, 280$ $\mathrm{mg} \cdot \mathrm{h} / \mathrm{L}$ after simulation of the reduction dose of $50 / 25 \mathrm{mg}$ and dose escalating of $70 / 70 \mathrm{mg}, 100 / 100 \mathrm{mg}$.

Supplementary Figure $3 \mathrm{~A}, 3 \mathrm{~B}$ and $3 \mathrm{C}$ showed the relationship between $C_{\max }, C_{\min }$ AUC, CL and BW with caspofungin $70 / 50 \mathrm{mg}$ in general patients and ICU patients and 70/35 mg in moderate $\mathrm{HI}$ patients, respectively. We find that $C_{\min }$ were constant in all investigated virtual patients and did not correlate to $\mathrm{BW}$ or other covariates. This was also valid for the CL. But $C_{\max }$ and AUC began a slow decline with the increase of body weight. The variation tendency was not obvious in $\mathrm{HI}$ patients.

As shown in Supplementary Figure 3D, patients with different ages showed no statistical difference in PK parameters. Compared with Westerner, $C_{\max }$ in Asian showed a slight increase and $C_{\min }$ showed a slight decrease in all of these general patients, ICU patients and HI patients (Supplementary Figure 4).

\section{Optimization of the clinical dosage regimens}

The WB-PBPK model coupled with a MCS showed the currently recommended dosage regimens of caspofungin in all patients achieved CFR $\geq 90 \%$ for C. albicans and C. glabrata, but achieved CFR $\leq 65 \%$ for $C$. parapsilosis, a maintenance dose of 100/100 mg in ICU and mild HI patients and 70/70 mg in moderate HI patients achieved CFR $\geq 90 \%$ for C. parapsilosis (Figure 4 and Table 4 ).

\section{Disscussion}

Drug research based on the mathematical models becomes extremely important for the optimization of clinical dosage regimens in special populations, mostly by providing rea- 
A

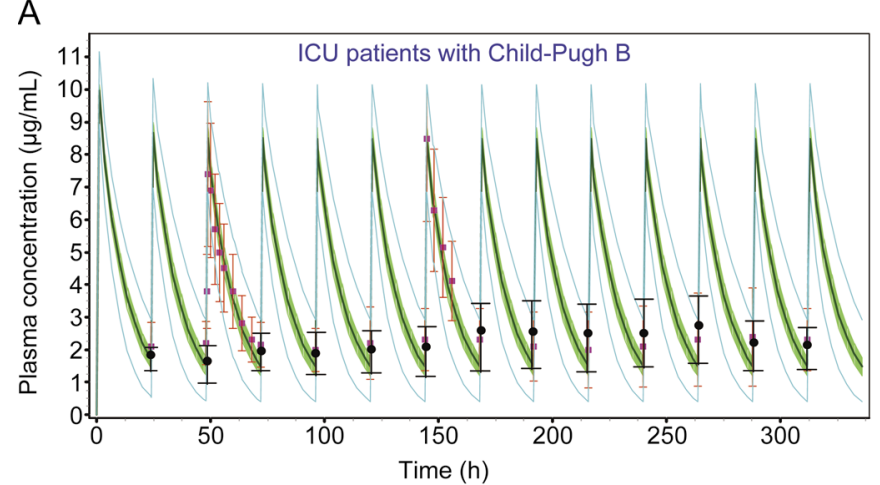

C

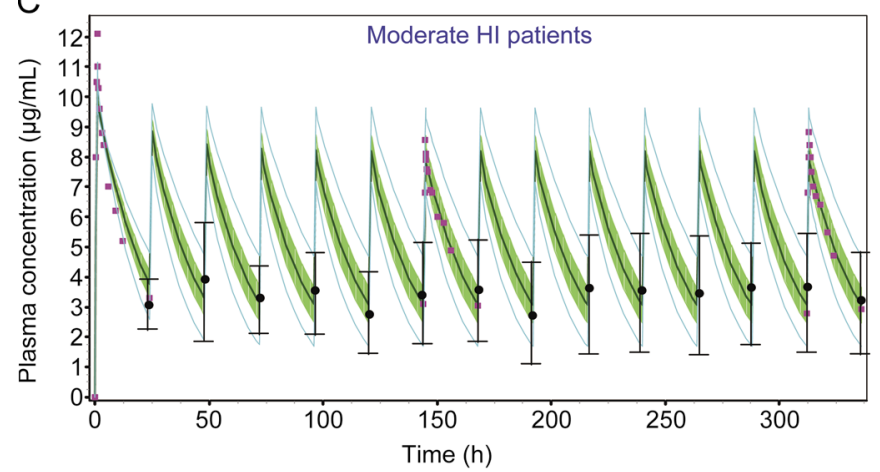

B

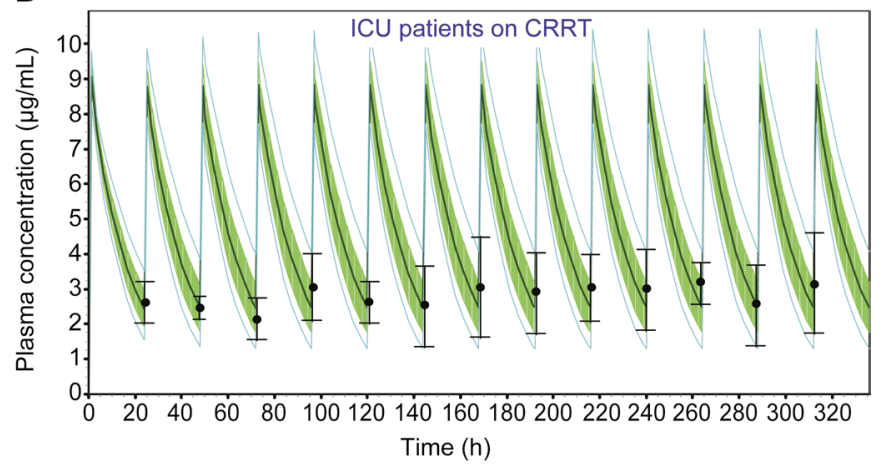

D

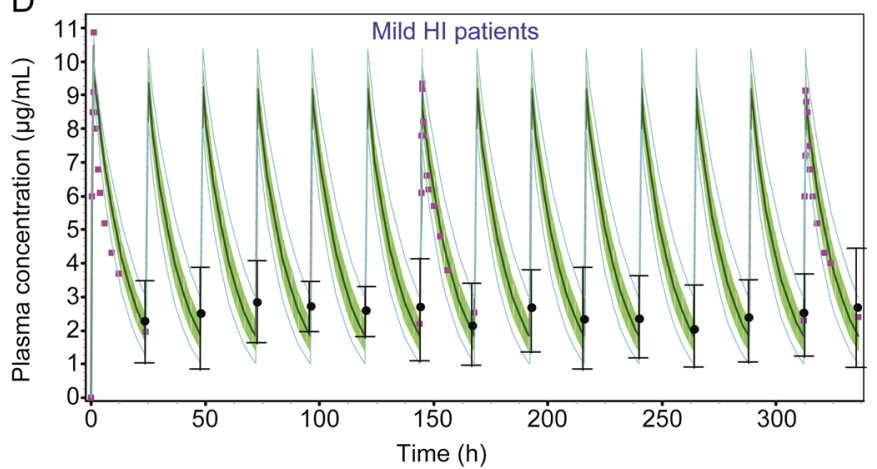

Figure 3. Simulated and mean observed (symbol \pm SD) plasma concentration-time profiles of caspofungin after the recommended intravenous multiple doses in ICU patients with Child-Pugh $B(A)^{[9]}$, ICU patients on continuous renal replacement therapy $(C R R T)(B){ }^{[17]}$, moderate $(C)$ and mild $H I$ patients $(D)^{[10]}$. A solid thick line adjacent to the middle of the concentration-time profile represents the mean of the predictive values. Solid squares represent the observed clinical concentration-time data ${ }^{[9,17,10]}$, and solid circles represent our data of trough concentrations (mean \pm SD). The shaded area represents the $90 \%$ confidence interval for the simulated data, and the thin lines on either hand represent individual simulated results of the $100 \%$ range of simulated individual data.

sonable dose selection method and thereby offer a reliable way to evaluate the risks and benefits. At present, WB-PBPK models have been used for prediction of the pharmacokinetics in special populations for their strength in data integration and delivery of mechanistic insights and superior predictive power ${ }^{[24]}$. These models could explain phenomenons from the perspective of mathematical model principles and can be applied to forecast disease dependencies and can also be used to investigate the variability expected in different patient populations ${ }^{[24]}$. Based on the WB-PBPK models, this investigation focus on the influence of disease state on the PK of caspofungin. But most importantly, the study makes innovation in combining WB-PBPK model with MCS to optimize the clinical dosage regimens in special patients.

This article first describes the use of WB-PBPK modelling to assist research and clinical study of PK of caspofungin in ICU patients and HI patients. The approach which integrated drug-specific parameters such as $\log P, \mathrm{p} K_{\mathrm{a}}$, solubility and permeability and in vitro data such as plasma protein binding and blood-to-plasma concentration ratio was able to simulate the pharmacokinetics of caspofungin across multiple dose levels in different human populations with suitable accuracy. The methodology also provided a reliable way to understand the mechanisms underlying the pharmacokinetic processes of caspofungin considering the influence of weight, age and race. Based on the changes of caspofungin pharmacokinetic parameters and combined with MCS, an optimization of dosage regimens was developed in these special patients.

After WB-PBPK simulation, we find that for ICU patients on CRRT, the PK parameters from the WB-PBPK simulation were in good agreement with those of the observed (our research data). When combined with MCS method, the recommended dosage regimen $(70 / 50 \mathrm{mg})$ is appropriate in these patients. But caspofungin maintenance dose should not be reduced to $35 \mathrm{mg}$ in ICU patients with Child-Pugh B which based on the Child-Pugh score if this classification is driven by hypoalbuminemia as it results in significantly lower exposure. A dosage reduction to $35 \mathrm{mg}$ daily following the $70 \mathrm{mg}$ loading dose is reasonable for moderate $\mathrm{HI}$ patients with Child-Pugh score B. If the maintenance dose was reduced to $25 \mathrm{mg} / \mathrm{d}, C_{\max }$ will decrease (Mean: $5.5 \mathrm{mg} / \mathrm{L}$, data were from the model simulation), which would affect the therapeutic effect of caspofungin. On the other hand, a maintenance dose increased to 50 $\mathrm{mg} / \mathrm{d}$ will lead to a higher $C_{\min }$ of $4.2 \mathrm{mg} / \mathrm{L}$ (data were from the model simulation), which would induce adverse reactions during the period of treatment.

With the increase of BW, PK of echinocandins might be altered due to changes in volume of distribution and/or CL. 

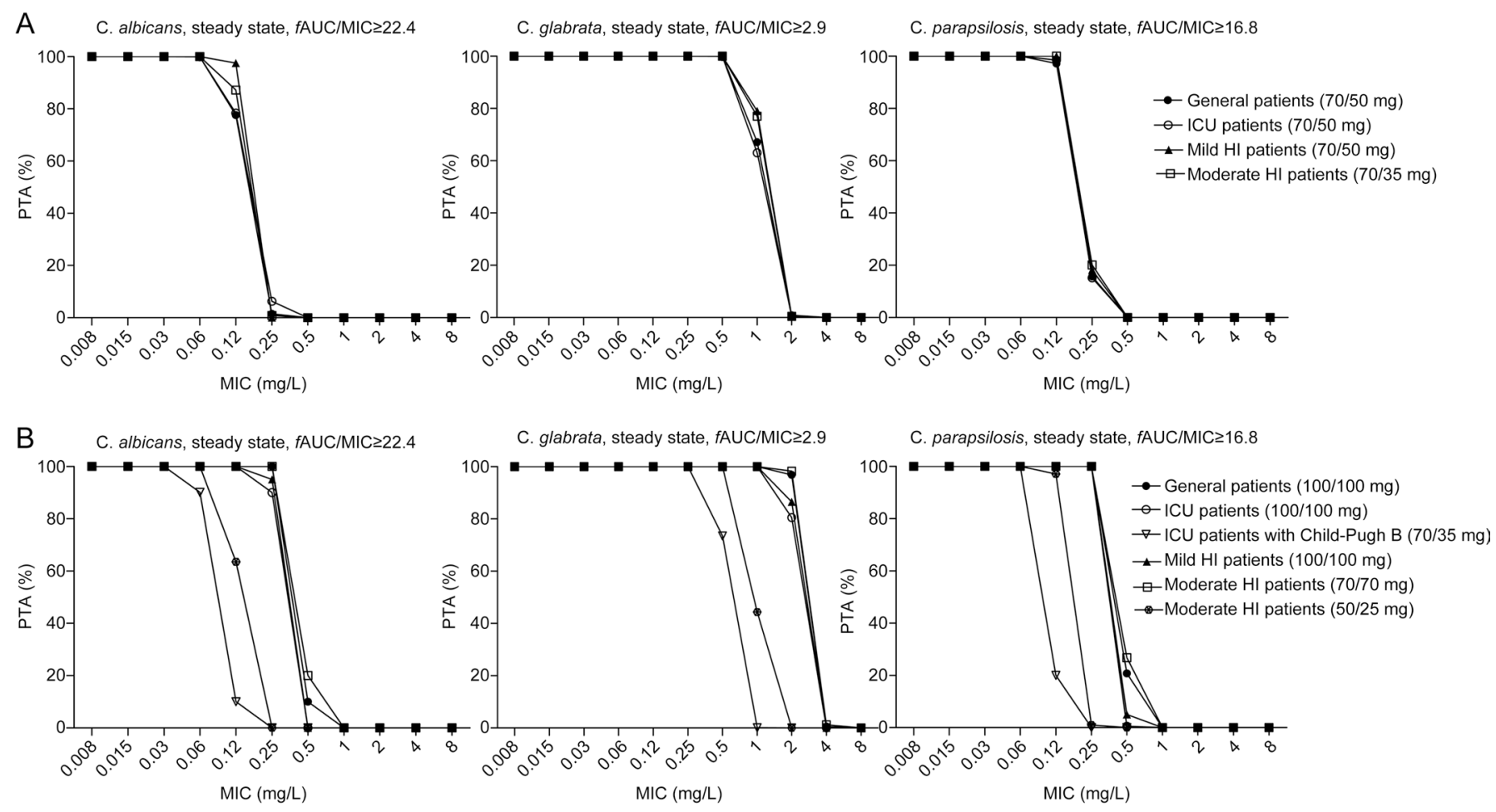

Figure 4. Figure 4A were PTA for C.albicans, C.glabrata, C.parapsilosis of the recommended dosage regimens of caspofungin in general patients, intensive care unit (ICU) patients (including ICU patients on CRRT and ICU patients with Child-Pugh B), mild hepatic insufficiency (HI) and moderate HI patients. The recommended dosage regimens of caspofungin for general patients (solid circles), ICU patients (hollow circles) and mild HI patients (solid triangles) were $70 / 50 \mathrm{mg}$, and for moderate $\mathrm{HI}$ patients (hollow squares), the recommended dosage regimen was 70/50 mg. Figure 4B were PTA for C. albicans, C. glabrata, C. parapsilosis of the adjustive dosage regimens of caspofungin in general patients (100/100 mg, solid circles), ICU patients (100/100 mg, hollow circles), ICU patients with Child-Pugh B (70/35 mg, downward hollow triangles), mild HI (100/100 mg, upward solid triangles) and moderate $\mathrm{HI}$ (70/70 mg, hollow squares; 50/25 mg, a circle with a fork in it) patients. The pharmacodynamic index was fAUC/MIC.

Studies have shown that PK in obese patients might be altered due to a change of more fat tissue in relation to $\mathrm{BW}$, change in liver metabolism and an increase in CL, and change in plasma protein constituents should also be considered ${ }^{[25-27]}$. Clinically, PK data of echinocandins in obese patients are scarce. One study demonstrated that patients weighing $>80 \mathrm{~kg}$ are advised to receive caspofungin $70 / 70 \mathrm{mg}^{[28]}$. In surgical ICU patients, weighing $>75 \mathrm{~kg}$ were advised to receive a dosage regimen of $70 / 70 \mathrm{mg}$ for lower $C_{\min }$ (decreased by $28 \%$ ) were predicted in these patients ${ }^{[7]}$. But in general ICU populations and hemato- poietic stem cell transplantation patients (BW: range 50-99 kg) were discovered have no effect on caspofungin $\mathrm{PK}^{[9,12]}$. With the WB-PBPK simulation, our study demonstrated that general and ICU patients weighing $>70 \mathrm{~kg}$ are advised to receive $70 / 70 \mathrm{mg}$. But no dose adjustment is needed for mild and moderate $\mathrm{HI}$ patients weighing $>70 \mathrm{~kg}$ for the recommended dosage regimens $(70 / 50 \mathrm{mg}$ for mild HI patients and $70 / 35 \mathrm{mg}$ for moderate HI patients) could achieve CFR $\geq 90 \%$ for most Candida spp., such as $C$. albicans and C. glabrata. For adult patients, age has no effect on the PK of all these patients, there

Table 4. Cumulative fraction of response \% (CFR \%) of caspofungin against Candida spp. in three different kinds of people.

\begin{tabular}{|c|c|c|c|c|c|c|c|c|c|c|c|c|}
\hline \multirow{2}{*}{$\begin{array}{l}\text { Dosing regimens } \\
(\mathrm{mg} / \mathrm{d})^{\mathrm{a}}\end{array}$} & \multicolumn{4}{|c|}{ C. albicans } & \multicolumn{4}{|c|}{ C. glabrata } & \multicolumn{4}{|c|}{ C. parapsilosis } \\
\hline & $\begin{array}{l}\text { General } \\
\text { patients }\end{array}$ & $\begin{array}{l}\text { ICU } \\
\text { patients }\end{array}$ & $\begin{array}{l}\text { Mild } \\
\mathrm{HI}\end{array}$ & $\begin{array}{l}\text { Moderate } \\
\mathrm{HI}\end{array}$ & $\begin{array}{l}\text { General } \\
\text { patients }\end{array}$ & $\begin{array}{l}\text { ICU } \\
\text { patients }\end{array}$ & $\begin{array}{l}\text { Mild } \\
\mathrm{HI}\end{array}$ & $\begin{array}{l}\text { Moderate } \\
\mathrm{HI}\end{array}$ & $\begin{array}{l}\text { General } \\
\text { patients }\end{array}$ & $\begin{array}{l}\text { ICU } \\
\text { patients }\end{array}$ & $\begin{array}{l}\text { Mild } \\
\mathrm{HI}\end{array}$ & $\begin{array}{l}\text { Moderate } \\
\mathrm{HI}\end{array}$ \\
\hline $70 / 50 \mathrm{mg}$ & 96.3 & 96.8 & 97.9 & - & 99.5 & 99.4 & 99.5 & - & 67.3 & 66.5 & 68.9 & - \\
\hline $70 / 35 \mathrm{mg}$ & - & 86.9 & - & 98.2 & - & 98.8 & - & 99.5 & - & 20.9 & - & 69.3 \\
\hline $50 / 25 \mathrm{mg}$ & - & - & - & 93.3 & - & - & - & 98.4 & - & - & - & 63.9 \\
\hline
\end{tabular}

${ }^{a}$ All dose regimens are virtual dosage regimens. 
is also no need for dose adjustment in old patients. Compared with Westerners, $C_{\max }$ in Japanese showed a slight increase and $C_{\text {min }}$ showed a slight decrease in all of these general patients, ICU patients and HI patients (Supplementary Figure 4). But no dose adjustment is needed.

WB-PBPK model coupled with MCS showed that the current recommended dosage regimens of caspofungin in all patients achieved CFR $\geq 90 \%$ for C. albicans and C. glabrata, but achieved $\mathrm{CFR} \leq 65 \%$ for $C$. parapsilosis. The dosage regimens of $100 / 100 \mathrm{mg}$ in ICU and mild HI patients and 70/70 $\mathrm{mg}$ in moderate $\mathrm{HI}$ patients achieved CFR $\geq 90 \%$ (Figure 4 ). So for C. albicans and C. glabrata in all patients, the recommended dosage regimens are reasonable. For $C$. parapsilosis, dosage regimens of 100/100 $\mathrm{mg}$ in ICU and mild HI patients and 70/70 $\mathrm{mg}$ in moderate $\mathrm{HI}$ patients are needed.

Assignment of metabolism to transporters in the model is not considered in this paper but would be necessary for more detailed conclusions about the sources of variability in caspofungin PK. This would also require careful consideration of the demographics of the studied population and of other sources of variability. One study demonstrated that the intake approach of caspofungin into the liver is a biphasic process with a fast, reversible binding to the surface of hepatocytes and a slow transport through the active OATP1B1 $1^{[5]}$. Clinically, effective and reliable data on transporters and enzymes from in vitro experiments are needed in WB-PBPK models ${ }^{[29]}$, but we did not obtain utilizable $K_{\mathrm{M}}$ and $V_{\max }$ values of OATP1B1 for the transport of caspofungin in the literature. So data of $V_{\max }$ and $K_{\mathrm{M}}$ in vitro is urgently needed to refine the WB-PBPK model of caspofungin. Another limitation of this study is that the data for the model verification were almost from the literate resources and we acquired them from the manuscripts, but not given by the original authors. In order to make up for the defects, we collected 508 trough concentrations from 161 patients (39 ICU patients with Child-Pugh B, 16 ICU patients on CRRT, 47 moderate HI patients and 68 mild HI patients) to evaluate the model and found that they all showed sufficient accuracy between predicted and observed data.

\section{Conclusion}

In conclusion, a WB-PBPK model for caspofungin was developed and qualified in diverse adult population groups. The model successfully predicted the intravenous and PK of caspofungin across multiple dose levels in normal human populations and special patients. This represents a case study that highlights the prospective applications of the WB-PBPK model combined with the MCS to provide clinical PK predictions and optimize the clinical dosage regimens. PK of caspofungin can be obtained before proceeding with clinical studies. Clinically, when BW is higher than $70 \mathrm{~kg}$ in ICU patients, an increasing maintenance dose of $70 \mathrm{mg}$ every day should be considered in humans at lower exposures. Dose reduction of caspofungin in ICU Patients with Child Pugh B will result in suboptimal exposure. In the future, combined with other methods, the model could be used as a basis for further clinical investiga- tions to enable a priori adjustment of drug dosing for maximal efficacy and minimal toxicity.

\section{Acknowledgements}

The authors gratefully thank Tangdu Hospital of Fourth Military Medical University for outstanding assistance in the software support (Gastroplus 9.0).

This work is supported by the National Natural Science Foundation of China (No 81473177 and 81672954) and the Shaanxi Provincial Natural Science Foundation (No 2016JM8015).

\section{Author contribution}

Ya-lin DONG designed the study and edited the manuscript; Qian-ting YANG contributed to the analysis, interpretation, manuscript writing, and final approval of the manuscript; Yajing ZHAI contributed to the conception and design, analysis, interpretation, manuscript writing, and final approval of the manuscript; Lu CHEN contributed to the data collection, analysis, interpretation, manuscript writing, and final approval of the manuscript; Tao ZHANG, Yan YAN, Ti MENG, Lei-chao LIU, Li-mei CHEN, Xue WANG contributed to the conception and design, data collection, interpretation, manuscript writing, and final approval of the manuscript; All authors read and approved the final manuscript.

\section{Supplementary information}

Supplementary information is available at the website of Acta Pharmacologica Sinica.

\section{References}

1 Pappas PG, Kauffman CA, Andes D, Benjamin DK, Calandra TF, Edwards JE, et al. Clinical practice guidelines for the management of candidiasis: 2009 update by the Infectious Diseases Society of America. Clin Infect Dis 2009; 48: 503-35.

2 Kofla G, Ruhnke M. Pharmacology and metabolism of anidulfungin, caspofungin and micafungin in the treatment of invasive candidosis: review of the literature. Eur J Med Res 2011; 16: 159-66.

3 Walsh TJ, Adamson PC, Seibel NL, Flynn PM, Neely MN, Schwartz C, et al. Pharmacokinetics, safety, and tolerability of caspofungin in children and adolescents. Antimicrob Agents Chemother 2005; 49: 4536-45.

4 Stone JA, Holland SD, Wickersham PJ, Sterrett A, Schwartz M, Bonfiglio $C$, et al. Single- and multiple-dose pharmacokinetics of caspofungin in healthy men. Antimicrob Agents Chemother 2002; 46: 739-45.

5 Sandhu P, Lee W, Xu X, Leake BF, Yamazaki M, Stone JA, et al. Hepatic uptake of the novel antifungal agent caspofungin. Drug Metab Dispos 2005; 33: 676-82.

6 Balani SK, Xu X, Arison BH, Silva MV, Gries A, DeLuna FA, et al. Metabolites of caspofungin acetate, a potent antifungal agent, in human plasma and urine. Drug Metab Dispos 2000; 28: 1274-8.

7 Nguyen TH, Hoppe-Tichy T, Geiss HK, Rastall AC, Swoboda S, Schmidt $J$, et al. Factors influencing caspofungin plasma concentrations in patients of a surgical intensive care unit. J Antimicrob Chemother 2007; 60: 100-6.

8 Smith BS, Yogaratnam D, Levasseur-Franklin KE, Forni A, Fong J. Introduction to drug pharmacokinetics in the critically ill patient. Chest 2012; 141: 1327-36. 
9 Muilwijk EW, Schouten JA, Leeuwen HJ, Zanten AR, Lange DW, Colbers A, et al. Pharmacokinetics of caspofungin in ICU patients. J Antimicrob Chemother 2014; 69: 3294-9.

10 Mistry GC, Migoya E, Deutsch PJ, Winchell G, Hesney M, Li S, et al. Single- and multiple-dose administration of caspofungin in patients with hepatic insufficiency: implications for safety and dosing recommendations. J Clin Pharmacol 2007; 47: 951-61.

11 Muilwijk EW, Lempers VJ, Burger DM, Warris A, Pickkers P, Aarnoutse RE, et al. Impact of special patient populations on the pharmacokinetics of echinocandins. Expert Rev Anti Infect Ther 2015; 13: 799-815.

12 Wurthwein G, Young C, Lanvers-Kaminsky C, Hempel G, Trame MN, Schwerdtfeger R, et al. Population pharmacokinetics of liposomal amphotericin B and caspofungin in allogeneic hematopoietic stem cell recipients. Antimicrob Agents Chemother 2012; 56: 536-43.

13 Sinnollareddy MG, Roberts JA, Lipman J, Akova M, Bassetti M, De Waele JJ, et al. Pharmacokinetic variability and exposures of fluconazole, anidulafungin, and caspofungin in intensive care unit patients: Data from multinational Defining Antibiotic Levels in Intensive care unit (DALI) patients Study. Crit Care 2015; 19: 33.

14 Rodriguez-Hernandez MJ, Ruiz-Perez de Pipaon M, Marquez-Solero M, Martin-Rico P, Caston-Osorio JJ, Guerrero-Sanchez FM, et al. [Candidemias: multicentre analysis in 16 hospitals in Andalusia (Spain)]. Enferm Infecc Microbiol Clin 2011; 29: 328-33.

15 Schmitt W, Willmann S. Physiology-based pharmacokinetic modeling: ready to be used. Drug Discov Today Technol 2004; 1: 449-56.

16 Wurthwein G, Cornely OA, Trame MN, Vehreschild JJ, Vehreschild MJGT, Farowski F, et al. Population pharmacokinetics of escalating doses of caspofungin in a phase II study of patients with invasive aspergillosis. Antimicrob Agents Chemother 2013; 57: 1664-71.

17 Weiler S, Seger C, Pfisterer H, Stienecke E, Stippler F, Welte R, et al. Pharmacokinetics of caspofungin in critically ill patients on continuous renal replacement therapy. Antimicrob Agents Chemother 2013; 57: 4053-7.

18 Jones HM, Dickins M, Youdim K, Gosset JR, Attkins NJ, Hay TL, et al. Application of PBPK modelling in drug discovery and development at Pfizer. Xenobiotica 2012; 42: 94-106.
19 Stader F, Wuerthwein G, Groll AH, Vehreschild JJ, Cornely OA, Hempel G. Physiology-based pharmacokinetics of caspofungin for adults and paediatrics. Pharm Res 2015; 32: 2029-37.

20 Andes D, Diekema DJ, Pfaller MA, Bohrmuller J, Marchillo K, Lepak A. In vivo comparison of the pharmacodynamic targets for echinocandin drugs against Candida species. Antimicrob Agents Chemother 2010; 54: 2497-506.

21 Jones HM, Parrott N, Jorga K, Lave T. A novel strategy for physiologically based predictions of human pharmacokinetics. Clin Pharmacokinet 2006; 45: 511-42.

22 Parrott N, Paquereau N, Coassolo P, Lave T. An evaluation of the utility of physiologically based models of pharmacokinetics in early drug discovery. J Pharm Sci 2005; 94: 2327-43.

23 Yang Q, Wang T, Xie J, Wang Y, Zheng X, Chen L, et al. Pharmacokinetic/ pharmacodynamic adequacy of echinocandins against Candida spp in intensive care unit patients and general patient populations. Int J Antimicrob Agents 2016; 47: 397-402.

24 Strougo A, Yassen A, Krauwinkel W, Danhof M, Freijer J. A semiphysiological population model for prediction of the pharmacokinetics of drugs under liver and renal disease conditions. Drug Metab Dispos 2011; 39: 1278-87.

25 Brill MJ, Diepstraten J, Rongen A, Kralingen S, Anker JN, Knibbe CA. Impact of obesity on drug metabolism and elimination in adults and children. Clin Pharmacokinet 2012; 51: 277-304.

26 Jain R, Chung SM, Jain L, Khurana M, Lau SW, Lee JE, et al. Implications of obesity for drug therapy: limitations and challenges. Clin Pharmacol Ther 2011; 90: 77-89.

27 Knibbe CA, Brill MJ, Rongen A, Diepstraten J, Graaf PH, Danhof M. Drug disposition in obesity: toward evidence-based dosing. Annu Rev Pharmacol Toxicol 2015; 55: 149-67.

28 Vorwerk CK, Tuchen S, Streit F, Binder L, Hofmuller W, BehrensBaumann W. Aqueous humor concentrations of topically administered caspofungin in rabbits. Ophthalmic Res 2009; 41: 102-5.

29 Edginton AN, Theil FP, Schmitt W, Willmann S. Whole body physiologically-based pharmacokinetic models: their use in clinical drug development. Expert Opin Drug Metab Toxicol 2008; 4: 114352. 